\title{
A Research Platform using Active Local Cooling directed at Minimizing the Blood Flow in Human Fingers
}

\author{
Jan Steckel \\ University of Antwerp \\ Centre for Healthcare Technology \\ Universiteitsplein 1, B-2610 Wilrijk \\ Belgium \\ Jan.Steckel@ua.ac.be
}

\author{
Frank Goethijn, Guido De Bruyne, \\ Vincent Nulens, Daniel Lacko, \\ Samuel Bey and Stijn Verwulgen, \\ University of Antwerp \\ Design Sciences - Productontwikkeling \\ Ambtmanstraat 1, B-2000 Antwerpen \\ Belgium
}

\begin{abstract}
Patients undergoing chemotherapy for the treatment of cancer often suffer from deterioration of extremities, such as neuropathy and onycholysis, a condition which leads to the partial detachment of the nail from the nail bed. To prevent these deterioration, cryotherapy is often used during the application of chemotherapy by means of e.g. frozen gel gloves or socks or ice baths. While effective, current cryotherapy is often unpleasant and unpractical for the patients involved such that the therapy is not accepted by a significant part of the patients involved. Furthermore, the classical cryotherapies leave little room for researching the underlying prevention mechanism through active control of the applied temperatures. A crucial step is to direct applied temperatures at minimizing the blood flow for it is widely assumed that the efficacy of the therapy lies in local vasoconstriction induced by the applied cooling, limiting the blood flow to the fingers. This paper proposes a platform which allows researchers to investigate the effect of controlled cooling on the blood flow in fingers and nail bed on blood circulation at extremities. Preliminary results indicate that local cooling indeed can invoke local vasoconstriction, resulting in a decreased blood flow measured using Laser-Doppler flowmetry.

Index Terms-Local Cooling, Prevention of onycholysis, Peltier Cooling, PID Control
\end{abstract}

\section{INTRODUCTION}

Onycholysis is a condition causing the (partial) detachment of the nail from the nail bed, starting at its distal or lateral attachment. Patient undergoing chemotherapy used for cancer treatment often suffer from hair loss and deterioration of extremities such as onycholysis [1], [2]. Cryotherapy, using a frozen gel glove Elastogel ${ }^{\mathrm{TM}}$ for example, has been shown effective for the reduction of onycholysis through a multicenter study [3]. The prevention of onycholysis increases the quality of life of the patient, while the original cancer treatment still remains effective. However, discomfort and intolerance to the cold gloves occurs in part of the patient population [3], causing a significant amount of the patients to abort the cryotherapy [3]. Furthermore, the Elastogel ${ }^{\mathrm{TM}}$ or other therapies leave little room to control the temperature of the cooling applied to the patient, possibly reducing the efficacy of the prevention mechanism and enhance patient's comfort. However prior to the implementation of such active and directed colling system, further research on the relation between local cooling and blood circulation and how to minimize the latter is needed. We propose the use of an active cooling system consisting of peltier elements [4] or other active coldinducing devices to finely control the temperature applied to the patient. Furthermore, only local cooling on strategically chosen positions is applied which is aimed towards enhancing the acceptance of future cryotherapy.

\section{THE EFFECT OF APPLIED COLD ON HUMAN EXTREMITIES}

When cold temperatures are applied to human extremities, such as fingers or hands, the body reacts in order to keep the body temperature at an acceptable base level [5]. When cold is applied by submersing the extremity (for example the finger) in cold water, a cold induced vasoconstriction (CIVC) occurs. This temporarily reduces the flow of blood to the finger. After a while, the blood vessels start to dilate, causing an abrupt rise in blood flow. This effect was first discovered by Lewis in 1930, dubbed the "hunting reaction" [6]. More recently, this term has been coined Cold Induced Vasodilation (CIVD) [7]. This effect can be seen as a protection mechanism for the extremities in prevention of cold-induced tissue damages. This process is sketched in figure 1. A sudden drop of blood flow after the start of the cooling occurs due to vasoconstriction. Cold-induced vasodilation swiftly follows, and the cycle repeats itself. The figure is a sketched version from real blood flow data measured using a Laser Doppler flow meter, found for example in [8].

In the application of the prevention of deterioration of extremities, we hypothesize that this effect is disadvantageous to the efficacy of the treatment. As the blood flow rises to levels at least equally high as the base level, more blood, and therefore, docetaxel drugs are delivered to the blood vessels at the fingernail, possibly increasing the probability and degreed of deterioration. Therefore, we seek mechanisms that effectively reduce the amount of blood delivered to the fingernail by delaying or avoiding the CIVD response. 


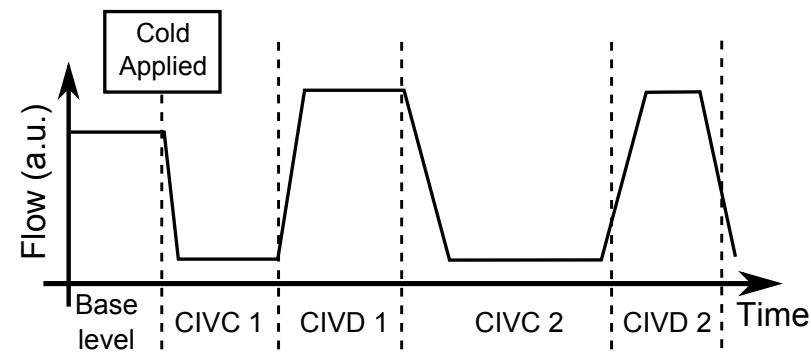

Fig. 1. Sketch of the bloodflow in the extremity when cold is applied. A first phase of vasoconstriction (labeled CIVC) is followed by a phase of vasoconstriction (labeled CIVD 1). After a while, the process repeats itself, with another cycle of vasoconstriction/vasodilation occurring. This effect is called the hunting response. The figure is a sketched version from real blood flow data measured using a Laser Doppler flow meter, detailed in [8].

One of the most common existing solutions, consisting of a cooled glove made from Elastogel, is currently being used as the de-facto standard for chemo-related cryotherapy. The glove is frozen for at least four hours at $-30^{\circ}$, and worn for a maximum of 60 minutes. After that time period, the glove is changed with a new frozen one, until the end of the administration of the chemotherapy drug. To estimate the temperatures that occur during the time the glove is worn, an experiment consisting of attaching five temperature sensors inside a glove was performed. The results of these measurements can be seen in figure 2. From the graph detailing the temperature evolution over time, it becomes clear that the temperature inside of the glove is all but constant, and that the temperature is only low during the first 15-20 minutes of application. This could be overcome by an active cooling system, which is capable of maintaining a low temperature over longer periods of time, with higher repeatability.

\section{Active Cooling System}

In order to overcome the limitations of the cooling approach of existing therapies, we propose to use an active cooling system based on Peltier elements. Figure 3 a) shows a sketch of the constructed systems. The cooling is applied on the side of the fingers, where the main arteries that supply the fingertip with blood are located. Two independent cooling zones allow flexible experimentation with different cooling schemes. Heat is transfered away to below the hand using heat pipes, which is then dissipated by a standard desktop computer CPU cooler equipped with a standard CPU fan.

The architecture of the system is detailed in figure 4. A PID (proportional-integral-derivative) controller controls the amount of electrical power delivered to the Peltier element, by means of an of-the-shelve proportional motor controller. The temperature on the finger skin is measured using an Negative Temperature Coefficient (NTC) temperature sensor, which is fed back to the PID controller, together with a desired temperature. Depending on the discrepancy between the desired temperature and the actual temperature, the output of the PID controller is set to reduce the error. The PID controllers run on an Arduino processing unit, which allows for swift development and adaptation of the controller software.
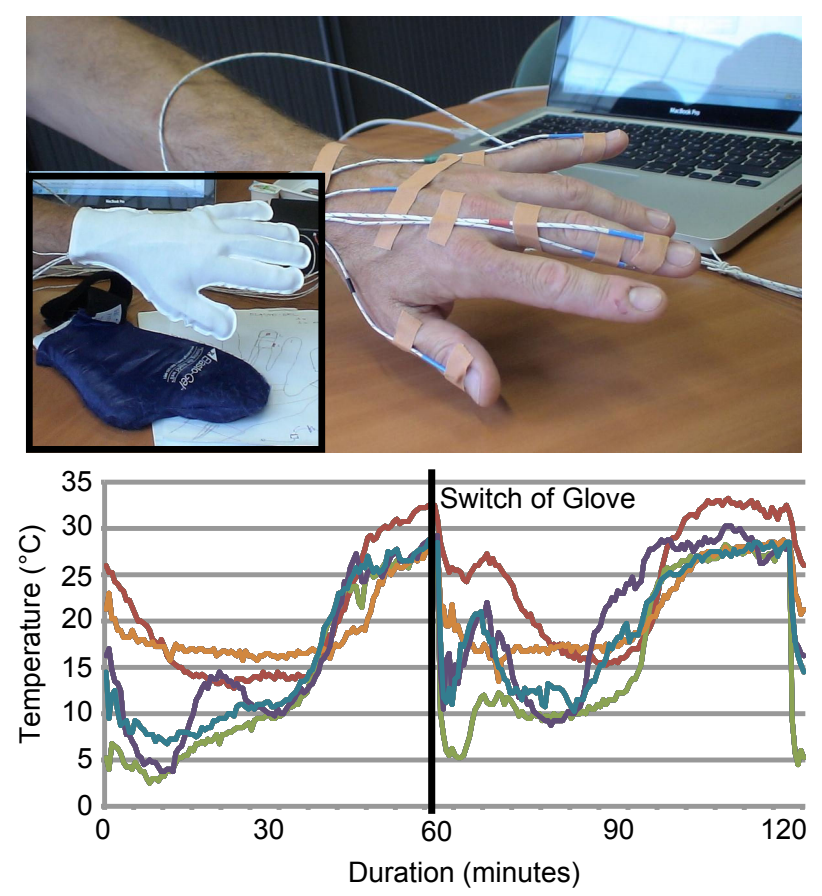

Fig. 2. Experiment using a frozen glove of Elastogel, with different temperature sensors attached to different positions of the hand of one of the authors (see picture). The grapth shows the evolution of the temperatures in function of time. Around minute 60 the glove was changed for a new one, mimicking the procedure occurring during real chemotherapy. The inset shows the instrumented hand inside the Elastogel glove.

The behavior of the control system can be seen in figure 5 a). A finger was placed inside the device, and a temperature profile consisting of a change in temperature from $14^{\circ}$ to $0^{\circ}$ in 5 minutes was applied. The resulting temperatures on the finger skin are displayed. As always, a trade off between reaction time and amount of overshoot exists, which can be altered by tweaking the system parameters of the PID controller. Figure $5 \mathrm{~b}$ ) shows the PID outputs in function of time. The motor controller is controlled by a servo-like input, which is actually a pulse-width modulated signal. A pulse width of $1.5 \mathrm{msec}$ equals to a neutral state, where the Peltier element is not driven. When the pulse width drops below 1.5 msec, the Peltier element is providing heat to the finger. A pulse width of more than $1.5 \mathrm{msec}$ results in a cooling action of the Peltier element.

\section{RESULTS}

In order to test the efficacy of the proposed system, we have measured the response of the blood flow in the index finger underneath the nailbed of using a Laser Doppler blood flow meter moorVMS-LDF2 by Moor Instruments [9] in three of the authors. The needle probe of the blood flow meter was positioned above the nailbed using a piece of foam. Using the prototype device, the right index finger of the test subjects was cooled to $4^{\circ}$, and the response of the blood flow was measured during 45 minutes. The blood flow meter outputs an analog voltage which is proportional to the instantaneous blood flow, which is measured using an arduino with a 


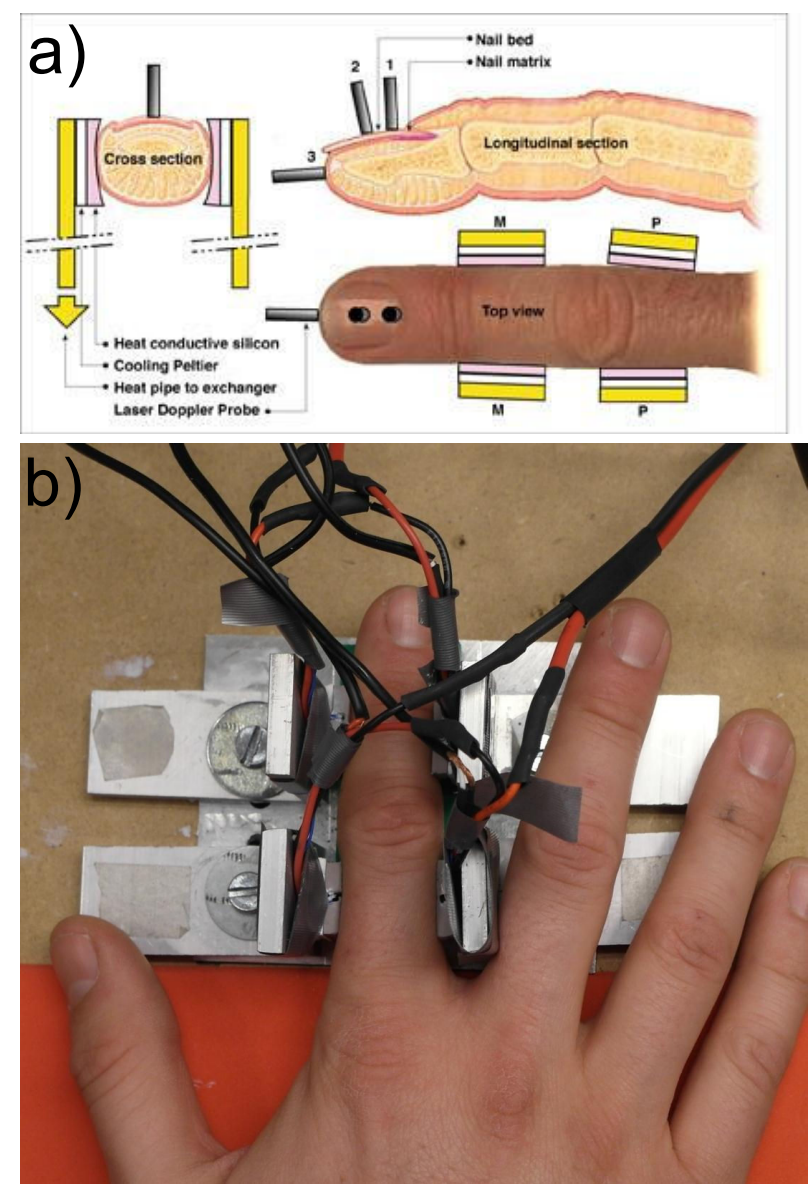

Fig. 3. a) Sketch of the local cooling system using Peltier elements. Peltier elements are being used to provide local cooling. Conductive silicon is attached to the Peltier element using heat conductive paste. The silicon provides good thermal contact between the Peltier element and the finger Heat pipes are used to transfer produced heat away from the fingers, allowing it to dissipate at another location. The NTC to measure the local temperature is embedded inside the heat conductive silicon. Numbers 1, 2 and 3 indicate interesting laser-doppler probe positions. During our experiments, probe position 2 was chosen. M stands for the Medial part of the finger, $\mathrm{P}$ stands for Proximal. The NTC element is mounted inside the conductive silicon layer b) shows a picture of the setup. The small holes inside the silicon are the locations of the NTC sensors.

sample rate of $50 \mathrm{~Hz}$. In post-processing, the resulting data was subsampled by a factor of 10 , and subsequently filtered using a median filter with a window length of 11 samples. Finally, a trend-line was fitted to the data by spline fitting and visual inspection. The resulting data can be seen in figure 6 . Strong vasoconstriction (indicated by CIVC) occurs in each of the three subject, indicating the efficacy of cooling locally. The measured circulation roughly linearly decreases with the applied temperature. The large peaks in the data originate from sudden movements by the test person, effectively moving the LDF probe causing the apparent sudden changes in blood flow values. During the experiments, no evidence of sudden vasodilatation was observed. We hypothesize that this is due to the less aggressive cooling regime when compared to classical cooling methods such as ice baths or frozen gloves. This, however, is to be examined in detail in follow-up studies.

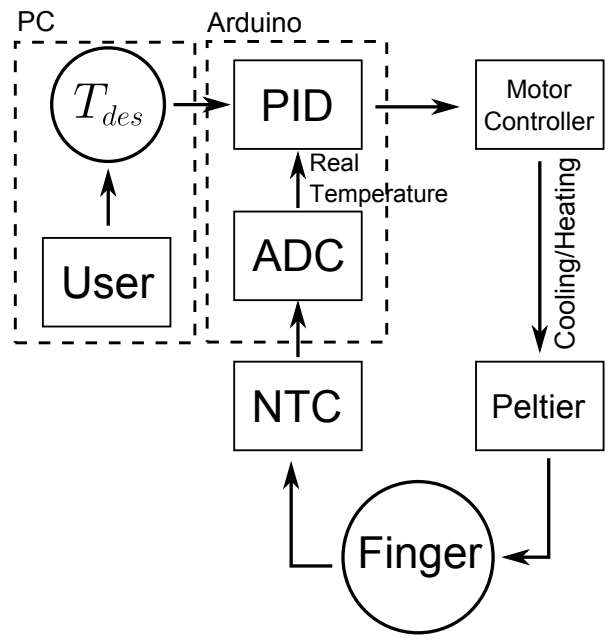

Fig. 4. Schematic overview of the control system. The desired temperatures can be set on a PC running Matlab, which outputs the data through a USB connection. A PID controller, implemented on an Arduino controls the electrical power sent to the Peltier element, which proportionally heats or cools the finger, until the desired temperature is reached. The temperature is measured using an NTC which is mounted in the thermally conductive silicon between the Peltier element and the finger, and converted from analog to digital by an AD-converter (ADC). The data from the PID controller and the NTC sensor is sent through the USB connection to the PC for further analysis. Two of these control systems are implemented to cool the medial and the proximal region of the finger independently.

Furthermore, none of the subjects reported severe discomfort.

\section{DISCUSSION AND FUTURE WORK}

We have proposed a method to apply local cooling to human extremities, namely the finger, as a research platform to understand the mechanisms governing deterioration caused by chemotherapy in relation to cooling, blood circulation and cold-induced vasodilation. The system can be used to devise cooling schemes which delay or prevent the onset of CIVD, effectively minimizing the amount of blood (and presumably the amount of harmful substances such as docetaxel) which reaches the nail bed.

Despite the limited size of the experiment, we have shown that local cooling of the right and left flank of the medial and proximal phalanx of the finger can evoke vasoconstriction in the nailbed. These encouraging results pose an interesting base for further experiments using a larger group of subjects under more controlled circumstances, as a whole range of parameters impacts both CIVC and CIVD [7]. Future work will encompass the search for effective cooling schemes which eventually improve the prevention of deterioration such as onycholysis. Furthermore, the research is aimed at increasing patient comfort and treatment acceptance. A system which allows cooling of all five fingers of the hand is currently being developed, which would allow more extensive testing of different cooling schemes and the effect on the blood flow thereof. All these experiments will eventually lead experiments in a clinical setting where the efficacy of local cooling for the prevention of onycholysis is investigated. Finally, more detailed measurements of the real blood flow in the extremities 

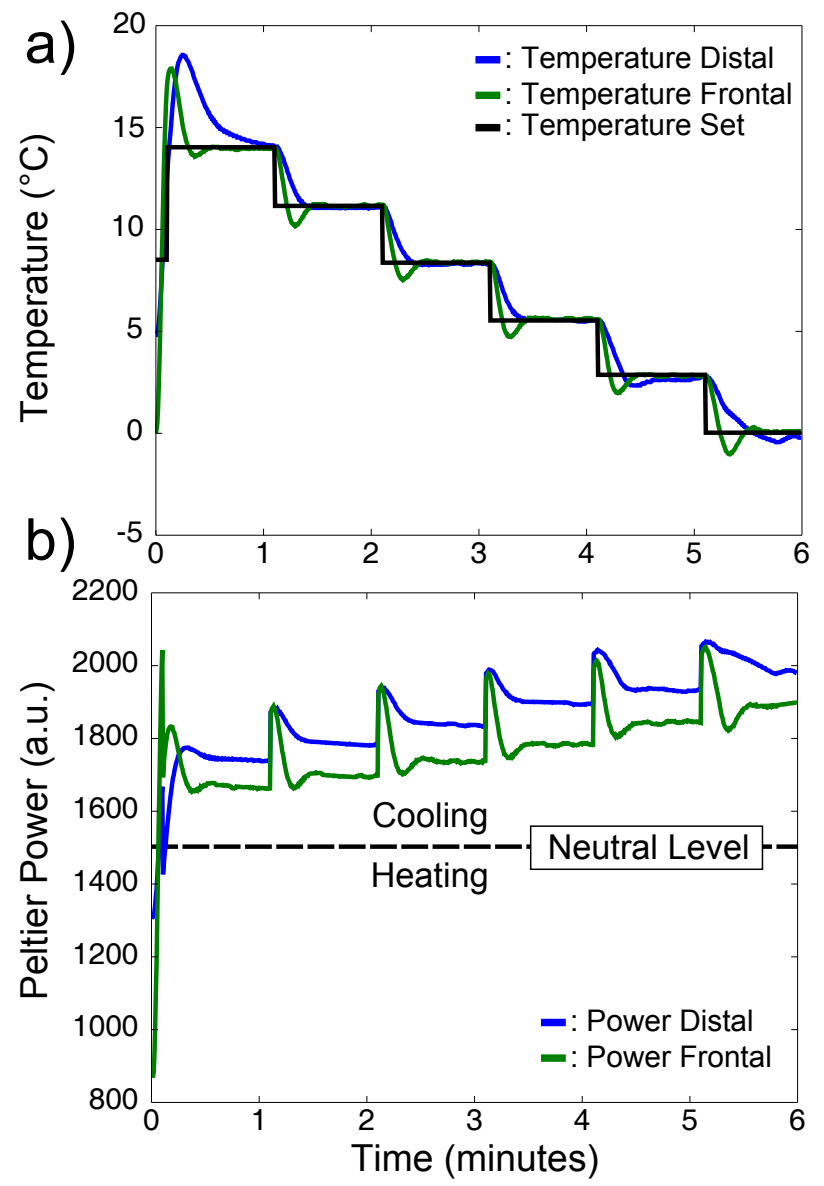

Fig. 5. a) Temperature controlling capabilities of the active cooling system. A right index finger was placed inside the device, and a temperature profile which consisted of stepping from $14^{\circ}$ to $0^{\circ}$ in 5 minutes was applied. The set temperatures are indicated by the black line. The green and blue line show the temperature responses of the system, whith the PID controller adjusting the cooling power delivered to the peltier elements. The system is able to control the skin temperature very accurately, and over long periods of time. b) the amount of power applied to the Peltier element in arbitrary units. A value of 1500 means no cooling or heating. A value between 1500 and 800 means the peltier element performs heating, a value between 1500 en 2200 means cooling is performed.

while wearing Elastogel gloves will be performed which enables us to set a more accurate baseline.

In the final design of the device, smart control mechanisms will be incorporated. At the moment, a cooling strategy based solely based on the finger temperature is used. However, the active cooling system also reports the current power that needs to be delivered to maintain the current set temperature. This can be used as a measure of vasodilation/vasoconstriction occurring, as the amount of heat transfered away from the peltier element will depend on the current blood flow. Furthermore, cheap and robust estimates of the current blood flow can be made using photoplethysmography. By using an Light Emitting Diode (LED) and a phototransisor, crude data about the current state of the blood vessels and blood flow can be extracted [10], [11]. In the final device, this could lead to blood-flow based cooling regimes, which could further increase the comfort, acceptance and efficacy of the treatment.
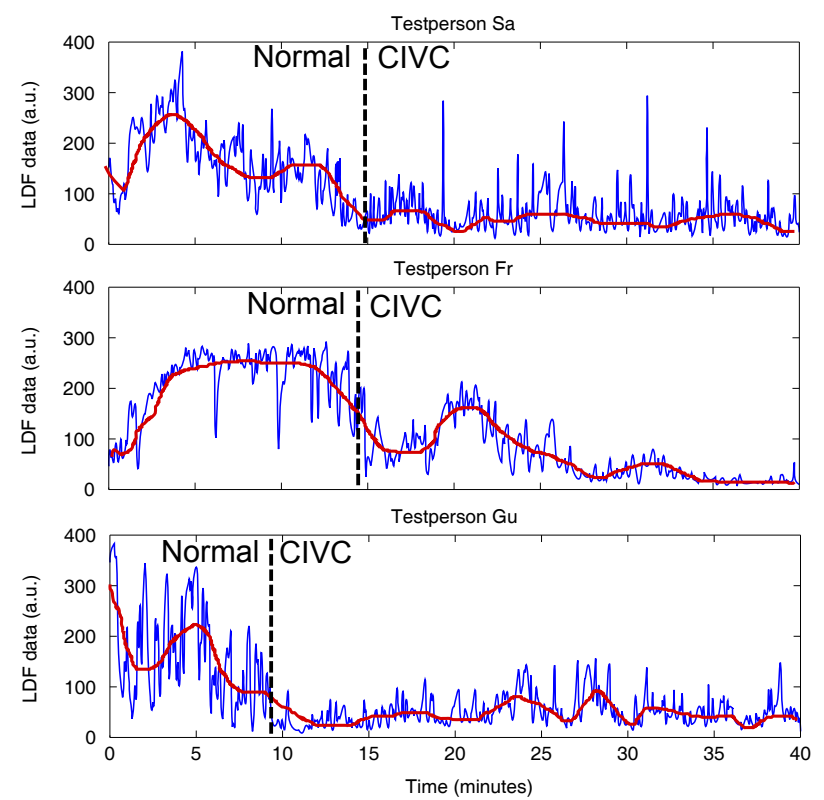

Fig. 6. Blood flow measured using Laser-Doppler flowmetry in function of time. The right index finger of the testsubject was cooled to $4^{\circ}$ measured at skin surface during the whole period, on three of the authors of the paper. In each of the tests strong vasoconstriction was observed after the cooling was applied (indicated by CIVC).

Finally, feedback of the blood flow measuring system and the cooling system could lead to a dosed delivery of chemotherapy drug based on the current state of blood flow, which could further increase the amount of protection against onycholysis.

\section{REFERENCES}

[1] S. Hussain, D. Anderson, M. Salvatti, B. Adamson, M. McManus, and A. Braverman, "Onycholysis as a complication of systemic chemotherapy," Cancer, vol. 88, no. 10, pp. 2367-2371, 2000.

[2] P. Gilbar, A. Hain, and V. Peereboom, "Nail toxicity induced by cancer chemotherapy," Journal of Oncology Pharmacy Practice, vol. 15, no. 3, pp. 143-155, 2009.

[3] F. Scotté, J. Tourani, E. Banu, M. Peyromaure, E. Levy, S. Marsan, E. Magherini, E. Fabre-Guillevin, J. Andrieu, and S. Oudard, "Multicenter study of a frozen glove to prevent docetaxel-induced onycholysis and cutaneous toxicity of the hand," Journal of clinical oncology, vol. 23, no. 19, pp. 4424-4429, 2005.

[4] V. Drebushchak, "The peltier effect," Journal of Thermal Analysis and Calorimetry, vol. 91, no. 1, pp. 311-315, 2008.

[5] N. Charkoudian, "Skin blood flow in adult human thermoregulation: how it works, when it does not, and why," in Mayo Clinic Proceedings, vol. 78, no. 5. Elsevier, 2003, pp. 603-612.

[6] T. Lewis, "Observations upon the reactions of the vessels of the human skin to cold," Heart, vol. 15, no. 177-208, p. 3, 1930.

[7] H. Daanen, "Finger cold-induced vasodilation: a review," European journal of applied physiology, vol. 89, no. 5, pp. 411-426, 2003.

[8] T. Bergersen, J. Hisdal, and L. Walløe, "Perfusion of the human finger during cold-induced vasodilatation," American Journal of PhysiologyRegulatory, Integrative and Comparative Physiology, vol. 276, no. 3, pp. R731-R737, 1999.

[9] "Moor instruments - laser doppler monitors," Jan. 2013. [Online]. Available: http://http://us.moor.co.uk/product/moorvms-ldflaser-doppler-monitor/1

[10] A. Kamal, J. Harness, G. Irving, and A. Mearns, "Skin photoplethysmography - a review," Computer methods and programs in biomedicine, vol. 28, no. 4, pp. 257-269, 1989.

[11] J. Allen, "Photoplethysmography and its application in clinical physiological measurement," Physiological measurement, vol. 28, no. 3, p. R1, 2007. 